\title{
Aplicación de técnicas multivariadas en el análisis del Clima Organizacional de una Municipalidad en Costa Rica
}

\author{
Application of multivariate techniques in the analysis of the organizational climate of a \\ municipality in Costa Rica
}

\author{
Ileana Morera Vargas \\ Universidad Nacional, Costa Rica, ileana.morera.vargas@una.cr
}

Alejandra Ávila Artavia
Universidad Nacional, Costa Rica, alejandra.avila.artavia@una.cr

Guillermo Zúñiga Arias

Universidad Nacional, Costa Rica,guillermo.zuniga.arias@una.cr

\begin{abstract}
Fecha de recepción: 26/12/2014 Fechas de reenvíos: 9/10/2015 - 30/11/2015, 21/04/2015 - 18/05/2015, Fecha de aceptación: 20/05/2016, Fecha de publicación: 27/07/2016.
\end{abstract}

\begin{abstract}
Resumen: Este artículo presenta un análisis; a partir de técnicas multivariadas; para determinar las variables prioritarias de intervención de un estudio de clima organizacional realizado en el 2013 en una Municipalidad en Costa Rica. El estudio inicial consistió en una descripción general de la percepción de variables que influyen en el clima organizacional de la misma, donde se analizaron 8 variables agrupadas en 3 dimensiones y recomendaciones para cada una de ellas.

Los resultados del análisis con técnicas multivariadas mostraron que la Municipalidad en estudio, si desea mejorar su clima debe priorizar y fortalecer acciones y recursos en las dimensiones de "Estructura, Procedimientos y Funciones" y "Motivación y Compromiso"; específicamente en las variables "Comunicación" y "Compromiso e Identidad". Además, debe considerar que los puestos de jefatura confieren mayor valor a la variable "Compromiso" que los otros puestos y el personal con más de cinco años de laborar en la institución a la variable "desafío y recompensa".

Se concluyó que el empleo de técnicas multivariadas muestra tendencias específicas que aportan al estudio inicial insumos para la definición de prioridades. En el caso de estudio los resultados del análisis multivariado indicaron que para el mejoramiento del clima organizacional, la Municipalidad debe orientarse, en primera instancia; a la planificación de estrategias para el mejoramiento organizacional según los hallazgos del análisis. Esto resulta fundamental para la definición y orientación presupuestaria e implementación de las acciones propuestas en el estudio primario.
\end{abstract}

Palabras Clave: desarrollo organizacional; clima organizacional; técnicas multivariadas; Municipalidad; planificación. 


\begin{abstract}
An analysis is conducted using multivariate techniques to determine the main intervention variables for an organizational climate study performed in 2013 in a municipality in Costa Rica. The initial study included an overview of the perception of variables influencing the organizational climate. Eight variables were analyzed and grouped in 3 dimensions, and recommendations were included for each of them.

According to the results of the multivariate technique analysis, in order to improve the municipality's climate, actions and resources in the "Structure, Procedures and Functions" and "Motivation and Commitment" dimensions should be prioritized and strengthened, specifically in the "Communication" and "Commitment and identity" variables. In addition, it should be considered that leadership positions give greater value to the "Commitment" variable than other positions, and personnel working in the institution for over five years give greater value to the "challenge and reward" variable.

It was concluded that the use of multivariate techniques show specific trends that provide the initial study with input to define priorities. In this study the results of the multivariate analysis indicated that in order to improve organizational climate, the municipality should first focus on planning strategies for organizational improvement. This is fundamental for the definition and budgetary guidance and implementation of the actions proposed in the primary study.
\end{abstract}

Keywords: organizational development; organizational climate; multivariate techniques; municipality; planning.

\title{
Introducción
}

De acuerdo con Koontz, Weihrich y Cannice (2012) el reto en la administración es diseñar y mantener un ambiente adecuado para que los colaboradores cumplan las metas de una manera eficiente. Señalan que un clima organizacional favorable genera compromiso y pertenencia, incrementa la motivación y se traduce en mayor desempeño elevando así la productividad. De esta forma, conocer la percepción de los funcionarios(as) acerca de las diversas condiciones laborales en las que se desenvuelven es vital para la sostenibilidad de cualquier entidad.

Según aproximaciones realizadas por Lewin, Lippit y White (1939), Schneider (1975), Brunet (1992) y Singh, Loncar (2010) el clima organizacional es un concepto multidimensional y, para algunos, se configura a partir de las características propias de cada organización. Estas se articulan en dimensiones que se gestan alrededor de características físicas estructurales, de procesos y de características de las personas de que la componen. Cardona y Zambrano (2014) partiendo de la revisión de diez instrumentos de evaluación del clima, recatan la coincidencia respecto a ocho dimensiones básicas: toma de decisiones, claridad organizacional, liderazgo, interacción social, motivación institucional, sistema de recompensas e incentivos, apertura organizacional y supervisión.

En la literatura hay un amplio número de estudios que muestran una relación positiva entre el clima y el desarrollo de la organización. Algunos recientes son el de Patlán-Pérez, Martínez 
y Hernández (2012) cuyo estudio concluye su asociación positiva con la satisfacción laboral; Katerndahl, Parchman y Wood, (2009) y Navarro-Reynoso, Hernández-Lamas, Robledo- Galván y Romero-Palacios (2008) asocian la satisfacción con variables propias del clima organizacional como la satisfacción con el salario y la carga laboral.

Chiang, Núñez, Martín y Salazar (2010), por su parte, refieren relaciones significativas; principalmente en hombres; entre clima y compromiso. En el estudio de Pérez-Zapata, Peralta Montecinos y Fernández-Dávila (2014) se describe la calidad de vida laboral y su relación con el clima organizacional, encontrando una relación significativa entre las mismas.

Dada la importancia de la relación observada entre el clima y el desarrollo organizacional, Amaro, González y Pérez (2015) anotan que su diagnóstico se ha constituido una técnica avanzada en la administración moderna en la búsqueda de mayor eficiencia empresarial, con el objetivo principal de detectar y evaluar las causas que afectan distintas variables en el desempeño de sus colaboradores(as). Estudiosos del tema como Litwin, Stringer, y Lickert se han interesado en los métodos para medir la percepción que los funcionarios(as) tienen sobre el clima y han sobresalido en el desarrollo de instrumentos para su valoración. El estudio de Cardona y Zambrano (2014), por su parte, aporta una revisión de 10 instrumentos con relación a sus propiedades psicométricas y las dimensiones evaluadas.

Para la medición del clima organizacional es frecuente el uso de instrumentos como los cuestionarios, apoyados por entrevistas y grupos focales. El clima de cada organización responde a una mezcla de características propias, por lo que la estructura de los instrumentos de medición empleados, las dimensiones y variables a analizar deben ser adaptadas a cada realidad. Los resultados permiten realizar un análisis descriptivo de las percepciones y valorar posibles acciones en la mejora del clima organizacional.

El análisis multivariante, permite describir e interpretar los datos provenientes de la observación de varias variables estadísticas, estudiadas conjuntamente con la finalidad de estudiar las relaciones que hay entre más de dos variables y buscar posibles maneras de organizar los datos (Cuadras, 2014). En este artículo se utilizaron técnicas multivariadas con la finalidad de aportar al estudio de Ávila y Morera (2013) en el diagnóstico, definiendo las variables prioritarias de acción para el mejoramiento del clima en una Municipalidad.

El objetivo del estudio original fue analizar la percepción de los funcionarios(as) de la Municipalidad de San Rafael de Heredia con respecto a variables clave para optimizar el desarrollo del clima organizacional. Se realizó un estudio exploratorio-descriptivo de tipo mixto de las dimensiones y variables seleccionadas sin establecer relaciones o prioridades y se proporcionaron recomendaciones generales que sirvieran como guía para definir acciones dentro de planes de mejoramiento del clima y procesos de planificación institucional. 
Tomando en cuenta la relevancia que tiene la percepción del clima en el desempeño de toda organización y de definir en los procesos de planificación y toma de decisiones, se consideró relevante indagar sobre las variables que impactan más la percepción de los funcionarios(as) en dicha entidad. Por lo tanto la pregunta de investigación fue determinar ¿cuáles variables impactan en mayor medida la percepción del clima organizacional en la Municipalidad de San Rafael de Heredia? Se buscó profundizar en la configuración propia del clima de la misma y ofrecer información que facilite la orientación de recursos y toma de decisiones hacia variables prioritarias de intervención.

\section{Marco conceptual}

La gran transformación económica, socialy tecnológica que se presenta durante la Revolución Industrial (segunda mitad del Siglo XVIII hacia inicios del Siglo XX) promueve una mayor atención en el estudio del trabajo y de las organizaciones como eje fundamental de desarrollo económico y social. FrederickTaylor, citado como el padre de la Administración Científica (1911), postula su enfoque en la racionalización del trabajo, teniendo como eje la labor individual y operación de tareas. Posteriormente, la Teoría Clásica (1916) liderada por Henry Fayol, visualiza la organización como un todo, más allá de la tarea individual, y se presta atención a las relaciones sociales y a la estructura organizacional, identificando roles que le dan un orden y valor funcional a los trabajadores. Es Elton Mayo, quien en 1924, lidera los estudios realizados en la Planta Hawthorne y concluye que una mejor productividad está relacionada a factores sociales y humanos. Surge así la Teoría de las Relaciones Humanas y del Comportamiento, que brinda un mayor protagonismo al individuo dentro de las organizaciones. Exponentes como Abraham Maslow, Peter Drucker, Harold Koontz y Ken Blanchard señalan la importancia del recurso humano en la organización como sistema (Jones, 2013; Koontz, Weihrich y Cannice, 2012).

La Teoría de las Relaciones Humanas identifica, dentro de los factores que se relacionan con la efectividad laboral: el sentido de pertenencia, la motivación, los valores, la dirección y la comunicación (Chiavenato, 2009). El comportamiento organizacional tendría así relación con el manejo y la atención que se preste a estos factores en el trabajo.

El enfoque administrativo de las Relaciones Humanas, que pone en primer plano a las personas, evoluciona cuando en la década de 1960 el mundo se encamina en la era del conocimiento y la globalización, en un entorno donde la constante es el cambio (Chiavenato, 2009). Ello promueve enfoques contemporáneos donde la organización funciona como un sistema que integra tanto elementos internos como externos a la organización (entorno). La figura del individuo es vista con mayor atención, posicionándola como el capital más importante y por lo tanto, se considera que invertir en él deriva en mayores niveles de desempeño. Es entonces cuando el clima laboral se convierte en un objeto de estudio en la valoración de estrategias de sostenibilidad y crecimiento organizacional, que involucra aspectos de monitoreo y calidad, mejoramiento continuo y fortalecimiento de la cultura organizacional. 
Estos nuevos enfoques de la administración enfatizan en el valor del recurso humano y están orientados a la alineación de la cultura ${ }^{1}$ y el clima organizacional como un factor crítico de éxito. Es decir, para el cumplimiento de los objetivos propuestos por una organización es indispensable que el modelo de gestión que se presume guiará la organización tenga una relación coherente con la percepción y accionar de quienes laboran en ella.

En concordancia con dichos enfoques cobra sentido el concepto de Comportamiento Organizacional (CO), comprendido como "el campo de estudio que investiga el efecto que los individuos, los grupos y la estructura tienen en la forma de actuar de la organización" (Franklin, y Krieger, 2011, p.11). Con el CO interactúan diferentes disciplinas de la conducta, las ciencias sociales y administrativas con el propósito del desempeño de ésta.

Según Franklin y Krieger, (2011), las organizaciones son “... entidades sociales coordinadas, que operan de manera deliberada para alcanzar objetivos específicos - tarea imposible de realizar por individuos que actuaran solos - a través de una estructura determinada" (2011. p. 2). Cada una posee una identidad única, caracterizada por dos elementos articulados entre sí:

1. La cultura organizacional es un modelo propio de presunciones (implícitas y explícitas, formales e informales) que son consideradas válidas y enseñadas a los nuevos colaboradores como la "forma adecuada" de pensar, sentir y actuar en esa organización particular.

2. El clima organizacional, comprendido como la interpretación, traducción y accionar de los colaboradores con relación a esa cultura en un momento determinado.

El clima describe las percepciones compartidas por los colaboradores de una organización en su entorno laboral, entendiéndolas como el proceso cognitivo (recibir, procesar e interpretar información) permitiéndole a una persona atribuir un significado particular a un conjunto de estímulos, partiendo de un esquema propio de creencias, valores y actitudes. Conocer esas percepciones, al fin de valorar el estado del clima y tomar decisiones para su mejora y aprovechamiento, es un reto organizacional. Segredo, (2012) lo define como el ambiente donde las personas hayan facilidades o dificultades para aumentar o disminuir su desempeño, la percepción que se tiene acerca de ellas y la influencia que ejercen las estructuras organizativas, factores internos o externos en el logro de objetivos.

Fischer (1992) cita que Litwin y Stringer (1968) realizaron estudios sobre el impacto del clima laboral en el rendimiento y demostraron que los mejores rendimientos se producen en organizaciones o ambientes con un clima que catalogan de excelencia (que estimula la creatividad e innovación) y de cooperación (trabajo en equipo). Campbell, Dunnette, Lawler y Weick (1970) señalan que

1 Traducida usualmente y de manera general en su misión, visión y valores, políticas y estructura. 
la autonomía, las remuneraciones, relaciones interpersonales y estructura influyen en el clima organizacional. Por otra parte, Likert (1961-1967) evidencia la importancia del liderazgo y estilos de supervisión en la definición de distintos climas y Halpin y Croft (1962) identifican aspectos asociados a la solidaridad, relaciones interpersonales, confianza, apoyo y dirección.

Parece claro entonces que la comprensión del clima de una organización se deriva de tres aspectos: psicológicos (comportamiento de las personas), de estructura de la organización y de la dinámica de los procesos internos. Podemos encontrar en la literatura dimensiones y variables estrechamente relacionadas con estos aspectos y de ahí que el estudio original partiera de esta fundamentación.

Varios investigadores han realizado aportes conceptuales y valoración de herramientas para medir el clima laboral. Dentro de los más reconocidos en el ámbito social y organizacional se encuentran: Halpin y Croft (1962), Likert (1967), Litwin y Stringer (1968) y Campbell, Dunnette, Lawler y Weick (1970). Sin embargo, de acuerdo con Cardona y Zambrano (2014), fueron Lewin, Lippit y White (1939) los pioneros en realizar las primeras aproximaciones al concepto de clima organizacional.

Los instrumentos más utilizados para medir el clima organizacional son los cuestionarios estructurados, pues con ellos se logra recopilar información acerca de la percepción que sobre las diferentes dimensiones seleccionadas puedan tener los colaboradores de una organización. Los resultados pueden ser apoyados además por entrevistas semiestructuradas y grupos focales.

Vale aclarar que cada organización tiene particularidades que las hace diferentes unas de otras, su clima responde a una mezcla de características propias a esta, por lo que la estructura de los cuestionarios, las escalas de medición y las dimensiones y variables que se analicen pueden ser adaptadas a cada realidad de acuerdo con las necesidades que visualicen. De ahí que los planteamientos realizados por diferentes autores se convierten en un marco referencial.

Los resultados que se derivan de aplicar un cuestionario y entrevistas semiestructuradas permiten realizar un análisis descriptivo de las percepciones y valorar posibles acciones en la mejora del clima organizacional. Este análisis puede generase, por un lado, a partir de técnicas univariadas y resaltar el efecto que tiene una sola dimensión sobre una variable dependiente; 0 en su defecto, utilizar técnicas multivariadas, que posibilita el análisis de la misma realidad pero utilizando varias variables al mismo tiempo. Esto permite una profundización del análisis pues facilita la compresión del fenómeno cuando son varias variables las que actúan directamente sobre el objeto de estudio (acercando al investigador con la realidad).

Cuadras, (2014, p.11), señala que "el Análisis Multivariante es un conjunto de métodos estadísticos y matemáticos, destinados a describir e interpretar los datos que provienen de 
la observación de varias variables estadísticas, estudiadas conjuntamente". Los métodos de análisis multivariable (también llamado multivariante) se utilizan así para estudiar las relaciones que hay entre más de dos variables y buscar posibles maneras de organizar los datos. Entre las técnicas más utilizadas se encuentran: Análisis de Varianza (ANOVA), Regresión Lineal, Análisis Discriminante, Análisis de Grupos.

Si se aplica el análisis multivariado al clima organizacional y este se ve como la variable dependiente, se puede decir que se generará un modelo que permita relacionar el clima organizacional con varias variables independientes que eventualmente impacten significativamente al clima organizacional.

En el estudio original realizado en la Municipalidad de San Rafael se analizaron los modelos de Likert (del enfoque tipológico) y el modelo de Litwin y Srtinger (del enfoque dimensional) con la finalidad de obtener puntos de congruencia que facilitaran la validación de las dimensiones y variables seleccionadas por líderes de la Municipalidad o, en su defecto, sugerir otras. Se seleccionaron tres dimensiones, ocho variables específicas e indicadores para cada una de ellas. Se seleccionaron a partir de revisiones teóricas, la experiencia de las profesionales a cargo del proyecto y análisis con el grupo gerencial de la Municipalidad de acuerdo a sus necesidades específicas. Las variables e indicadores fueron agrupadas acorde a características comunes que califican en cada dimensión. La Tabla1 permite visualizar su composición.

Tabla 1

Dimensiones, variables e indicadores analizados en el estudio original

\begin{tabular}{l|ll}
\hline Dimensiones & Variables & Indicadores $^{2}$ \\
\hline Relaciones Interpersonales & Relaciones horizontales & Confianza \\
& & Empatía \\
& & Transparencia \\
& & Cooperación \\
\cline { 2 - 3 } & Relaciones verticales & Cooperación \\
& & Confianza \\
& & Transparencia \\
\hline & & Justicia \\
\hline Estructura, Funcionamiento, & Comunicación & Información externa \\
Procesos & & Información interna \\
\hline
\end{tabular}




\begin{tabular}{|c|c|c|}
\hline Dimensiones & Variables & Indicadores $^{2}$ \\
\hline & \multirow{8}{*}{ Planificación y Coordinación } & Orientación para la ejecución de tareas \\
\hline & & Claridad del rumbo \\
\hline & & Participación \\
\hline & & Resultados \\
\hline & & Medios y recursos \\
\hline & & Coordinación y administración de labores \\
\hline & & Funciones acorde a capacidades \\
\hline & & Oportunidad de opinión y decisión \\
\hline \multirow{6}{*}{$\begin{array}{l}\text { Motivación y } \\
\text { Compromiso }\end{array}$} & Empoderamiento & Capacitación y crecimiento profesional \\
\hline & & Claridad del puesto y sus funciones \\
\hline & Compromiso & Percepción de pertenencia \\
\hline & e Identidad & $\begin{array}{l}\text { Importancia de su trabajo para logro de metas } \\
\text { institucionales }\end{array}$ \\
\hline & Desafío & Reto \\
\hline & Recompensa & Percepción de retribución \\
\hline
\end{tabular}

Fuente: elaboración propia con datos del estudio original.

A continuación se define cada una de las variables y dimensiones mencionadas. ${ }^{3}$

1. Relaciones Interpersonales (RI):

Consisten en la interacción recíproca entre dos o más personas. Están determinadas por aspectos intrínsecos (personalidad, valores, habilidades para la socialización, aspectos fisiológicos) y extrínsecos (reglas o leyes en contextos específicos, canales y/o medios de comunicación). En el entorno laboral, son el eslabón básico de flujo de información, facilitador de necesidades individuales, grupales e institucionales.

Las RI son valoradas en dos grandes variables: Relaciones Horizontales (RIH) y Relaciones Verticales (RIV), para conocer la percepción del impacto dentro de una estructura jerárquica y el nivel motivacional que al interior se gesta.

Se entiende por RIH el intercambio entre personas de un mismo rango o semejante en todos los niveles. Las RIV son aquellas que se gestan entre personas de niveles distintos, ya sea ascendente o descendente. La percepción de verticalidad u horizontalidad no siempre coincide con el

\footnotetext{
$2 \quad$ No se abordan en el presente

3 Estas se tomaron del estudio original.
} 
planteado en la estructura formal de la empresa (organigrama) sino en la percepción de los roles ejercidos, (a quién se percibe como compañero(a) o jefe(a)).

A partir de esta diferenciación, se abordaron indicadores propios de la dinámica interpersonal entre personas percibidas como pares, superiores o subalternos (ver tabla 1).

\section{Estructura - funcionamiento - procesos:}

Se refiere a la organización de cargos y responsabilidades que deben cumplir sus miembros (sistema de roles) con la finalidad de alcanzar las metas propuestas en el plan estratégico de empresa, Para ello se establecen procesos que sirvan de guía para la ejecución de las diferentes actividades y mecanismos de coordinación para un adecuado funcionamiento.

Su objetivo es lograr las metas planteadas en el proceso de planificación, donde es trascendental la participación,conocimientoyflujodeinformaciónquepermitaclaridadenlasfuncionesysurelación directa con los planes y logros alcanzados. Para ello se consideraron como variables de análisis ${ }^{4}$

la Comunicación, Planificación y coordinación, y Empoderamiento de las personas respecto a sus funciones.

3. Motivación y compromiso (Analizadas a partir de indicadores de Tabla 1):

La motivación se entiende "todo lo que impulsa a una persona a actuar, o a desear actuar de una determinada forma o, como predisposición de una persona a desarrollar un comportamiento orientado hacia una meta" Ricarte (SF). En el entorno laboral, este impulso se traduce en un esfuerzo para el logro de metas definidas. Esta puede ser intrínseca (causas internas) y extrínseca (causas externas).

La motivación y el compromiso se ven afectados por las otras dos dimensiones en análisis (Relaciones Interpersonales y la Estructura-Funcionamiento-Procesos), sin embargo, se rescataron como variables otros elementos que naturalmente pueden ser catalogados por los funcionarios como agentes motivadores. Ellos son: compromiso e identidad, desafío y recompensa. Los indicadores pueden observarse en la tabla 1.

Una vez indicadas las dimensiones, variables e indicadores utilizados en el estudio original, se presenta en la Figura 1 el curso a seguir en el presente estudio.

$4 \quad$ Factores críticos de éxito en el funcionamiento de toda estructura. 


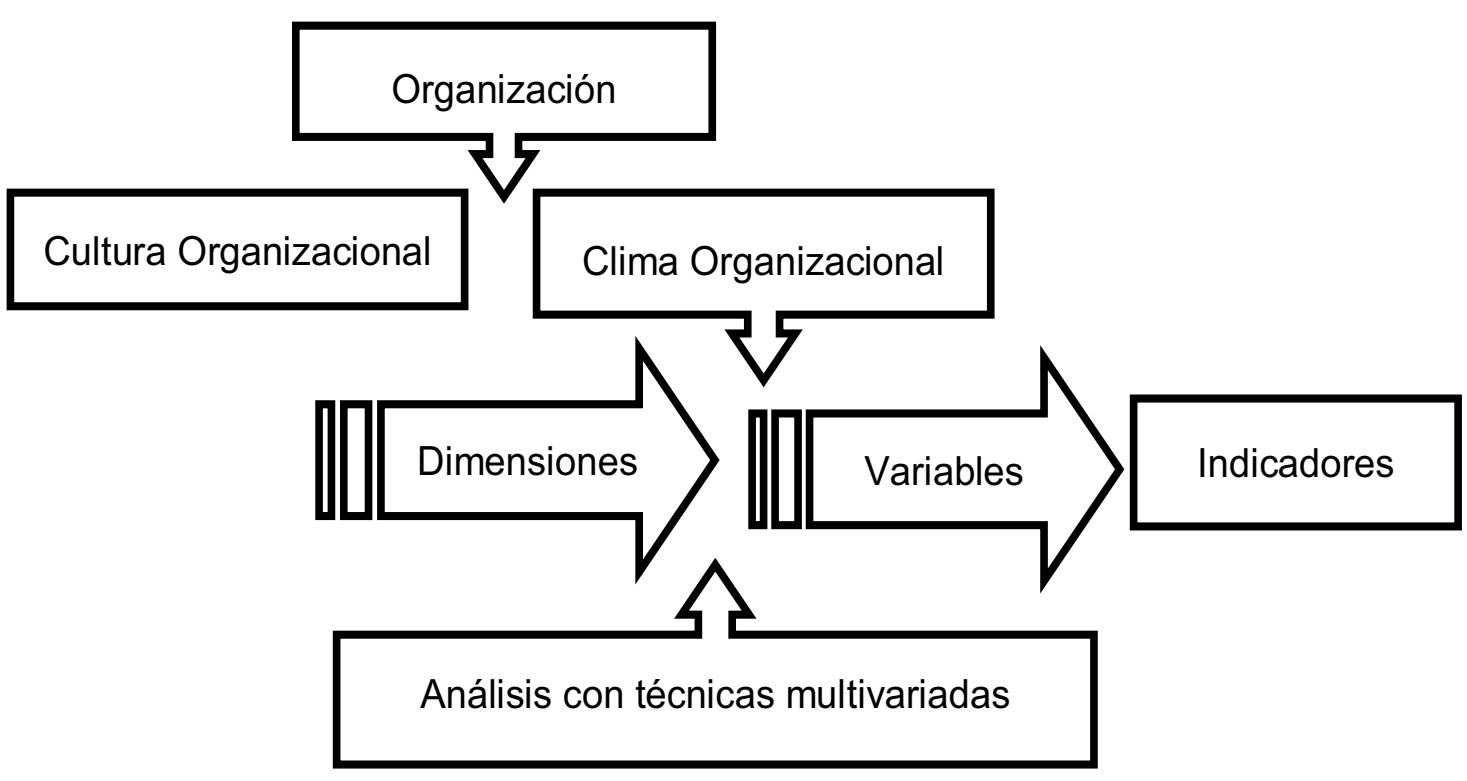

Figura 1. Curso del estudio. Fuente: elaboración propia con datos del estudio original.

\section{Metodología}

La presente investigación se basó en un estudio de la percepción de los funcionarios(as) de la Municipalidad de San Rafael de Heredia sobre variables que influyen en el clima organizacional en los meses d mayo a septiembre del 2013. Este fue realizado por el Programa CAMBIOS de la Escuela de Planificación y Promoción Social (EPPS) de la Universidad Nacional de Costa Rica (UNA).

El estudio fue exploratorio-descriptivo y de tipo mixto. No hay referencias a trabajos anteriores en la Municipalidad de San Rafael en el tema de clima organizacional lo que presenta una oportunidad de exploración y de mejoramiento de las actividades dentro de la gestión municipal.

En este se analizó la percepción con base en 3 dimensiones (relaciones interpersonales; estructura, procedimientos y procesos; y motivación y compromiso) divididas en 8 variables, a saber: relaciones interpersonales verticales, relaciones interpersonales horizontales, comunicación, planificación y coordinación, empoderamiento, compromiso e identidad, desafío y recompensa.

Para ello se aplicó un cuestionario al 100\% de la población (censo a 101 personas), una entrevista semiestructurada al 15\%, sesiones grupales (reuniones y talleres) además de revisión 
de fuente secundarias y observación no estructurada. Con base en los resultados se realizaron recomendaciones generales que sirvieran como guía para definir acciones dentro de los planes de mejoramiento y procesos de planificación institucional.

\section{Etapa cualitativa de la investigación}

En esta etapa se realizaron las entrevistas a los informantes claves y se trabajó con ellos para obtener la información vivencial del clima organizacional de la Municipalidad (observación, sesiones grupales y talleres). Esta información se sistematizó utilizando matrices por temas principales. La información sistematizada se utilizó como complemento de los resultados de los resultados del censo. La información cualitativa facilitó la comprensión de los resultados de la investigación cuantitativa.

\section{Etapa cuantitativa de la investigación}

Siendo un censo la técnica cuantitativa principal no se puede determinar un tamaño ni una confianza estadística. Además, no es necesario que se realice inferencia estadística de los resultados por ser el censo representativo de toda la población en estudio (funcionarios operativos y administrativos de la municipalidad).

El cuestionario del censo contó con 90 preguntas que contemplan aspectos relacionados con la tipología del encuestado y una segmentación en preguntas, con escala de Likert, para las ocho variables del estudio. El cuestionario se construyó con la articulación del marco conceptual utilizado por los investigadores para el desarrollo de este artículo. Se validó con la participación de expertos en clima organizacional y psicología de la Universidad de Costa Rica.

Se realizaron dos pruebas de campo. La primera a lo interno de la Escuela de Planificación y Promoción Social de la Universidad Nacional, con el objetivo de determinar la funcionalidad y operacionalidad de las preguntas, y los tiempos de aplicación. Esta se realizó con estudiantes y profesores de la Unidad Académica. Para la segunda validación, se realizaron una serie de aplicaciones a funcionarios de otras municipalidades con las que la Unidad Académica trabaja. Esta segunda prueba permitió determinar si las preguntas eran bien entendidas por los funcionarios y el tiempo real de aplicación por funcionario. Los resultados de ambas pruebas no fueron utilizados dentro del estudio.

En el análisis descriptivo cuantitativo se trabajó con porcentajes por dimensión, su relación con los años de trabajo y el nivel en la organización. 
Para este artículo la investigación es de tipo cuantitativa. Se empleó el procesador SPSS para los siguientes análisis:

- $\quad$ prueba de diferencia de medias independientes de las dimensiones

- análisis descriptivo de las variables

- diferencia de medias por puesto, nivel y tiempo laborado

- regresión logarítmica

Este estudio facilitó, con la utilización de estos métodos, la profundización en el análisis del clima organizacional permitiendo observar aquellas dimensiones y variables que determinan en mayor medida el clima de la Municipalidad de San Rafael.

\section{Diferencia de medias independientes}

Esta técnica permite determinar si existen diferencias entre grupos (control) y otras variables de interés, esta diferencia deberá ser significativa. En el caso de la investigación se determinó cuáles de las ocho variables de clima organizacional tienen diferencias significativas entre las variables de control en esta población particular. Entre sus ventajas, está que permite obtener diferencias entre grupos (significativas); pero tiene como limitante, que se deben de tener estos grupos de control definidos desde el inicio de la investigación.

En esta investigación se trabajó con una diferencia de medias independientes en la que se utilizó como variables control: 1) los años de trabajo en la organización y 2) el nivel administrativo y/o político que tiene el entrevistado.

\section{Descripción de datos}

En esta etapa del proceso se realizaron análisis para la búsqueda de tendencias, frecuencias, análisis descriptivos (tendencia central y de dispersión) y casos extremos en la base con la finalidad de conocer el comportamiento de los datos a investigar. El análisis descriptivo exploratorio tiene como principal ventaja que resulta simple de realizar y analizar, mas no favorece la profundización del análisis de los fenómenos en estudio. Adicionalmente, de la facilidad analítica, el porcentaje es un cálculo muy sensible a casos extremos por lo que se debe de tener en cuenta la homogeneidad de la variable para su cálculo. 


\section{Regresión logarítmica}

Esta técnica permite determinar elementos de causalidad. El modelo tiene una variable dicotómica $(0,1)$ dependiente y una o varias variables independientes. La ventaja e idea principal de ésta es medir el cambio porcentual entre una variable independiente y su impacto en la variable dependiente, es decir, se obtienen las variables independientes significativas y el impacto porcentual de estas en la variable dependiente. Su limitación es que se debe de tener la variable dependiente formulada como tal desde el inicio de la investigación.

Esta técnica es un complemento a la regresión múltiple. En muchos de los casos son técnicas similares (Hair et al., 1995).

\section{Resultados}

El artículo profundizó en el análisis de datos del estudio realizado por Ávila y Morera, (2013) empleando técnicas multivariadas con la finalidad de establecer tendencias que aporten a la priorización de acciones en los procesos de planificación. Las variables de interés se agruparon en tres categorías (igual que en el estudio original) para describir la percepción de los funcionarios(as): Percepción Favorable, Percepción Desfavorable y Percepción Nula o Indiferente.

Se realizó un análisis descriptivo (global e individual) de las dimensiones y variables, representados en forma de tablas y gráficos. Estos resultados fueron cruzados por el puesto del entrevistado (Gerencia y jefatura, Asistentes, Inspectores, Operarios, y otros) ${ }^{5}$. Las conclusiones se presentaron por dimensión de análisis.

\section{Test de Medias Independientes}

La Tabla 2 presenta el test de medias independientes de la percepción de agrado del clima organizacional y su impacto en las tres dimensiones de estudio. Los resultados muestran que las dimensiones "Estructura, procedimientos y funciones" y "Motivación y compromiso" presentan diferencias entre las personas que contestaron que les agrada o no les agrada el clima organizacional de su institución; indicando que el promedio de valoración de estas dimensiones es significativamente mayor en los sujetos que contestaron que no les agrada el clima actualmente. Esto indica que, cuando los funcionarios(as) refieren que no les agrada el clima, lo hacen en mayor medida refiriéndose a estas dimensiones. 
Tabla 2

Estadísticos de grupo y test de diferencia de medias independientes de las tres dimensiones en estudio.

\begin{tabular}{llcll}
\hline Dimensiones en estudio & Media & $\begin{array}{c}\text { Signo de la } \\
\text { diferencia }\end{array}$ & $\begin{array}{c}\text { Sig. Test de Medias } \\
\text { Independientes }\end{array}$ \\
\hline Relaciones Interpersonales & Me agrada & 3,27 & - & 0,447 \\
& No me agrada & 3,34 & & 0,489 \\
Estructura, Funciones y Procedimien- & Me agrada & 3,15 & - & $0,033^{* *}$ \\
tos & No me agrada & 3,29 & & $0,071^{*}$ \\
Motivación y Compromiso & Me agrada & 3,05 & - & $0,058^{*}$ \\
\cline { 2 - 3 } & No me agrada & 3,26 & & 0,181 \\
\hline
\end{tabular}

Nota: Significancia al* $90 \% ;{ }^{* *} 95 \%$; ${ }^{* *} 99 \%$

Fuente: Elaboración propia con datos del estudio original.

\section{Estadísticas Descriptivas}

Las estadísticas descriptivas principales (promedio, desviación estándar entre otros) de las ocho variables bajo análisis se muestran en la Tabla 3. Se observa cómo el coeficiente de variación es inferior a $20 \%$, determinando que la población bajo estudio se considera homogénea.

Por otra parte, la media de las dimensiones es cercana a 3, indicando que existe una tendencia a responder en el punto medio de la distribución. Las variables de "desafío y recompensa" son las que tienen las valoraciones menores, siempre orientadas a la tendencia central. 
Tabla 3

Estadísticas descriptivas de las variables de análisis. Estudio original sobre Análisis de la percepción de funcionarios y funcionarias de la Municipalidad de San Rafael de Heredia respecto a variables claves para optimizar el clima organizacional. Mayo - Setiembre 2013.

\begin{tabular}{lllc}
\hline Dimensiones & $\mathrm{N}$ & Media & Desv. típ. \\
\hline $\begin{array}{l}\text { Relaciones Interpersonales } \\
\text { Horizontales }\end{array}$ & 92 & 3,2533 &, 64030 \\
$\begin{array}{l}\text { Relaciones Interpersonales } \\
\text { Verticales }\end{array}$ & 92 & 3,1940 &, 62028 \\
$\begin{array}{l}\text { Comunicación } \\
\text { Planificación y Coordina- }\end{array}$ & 92 & 3,0304 &, 64752 \\
ción & 92 & 3,0890 &, 59087 \\
$\begin{array}{l}\text { Empoderamiento } \\
\text { Compromiso }\end{array}$ & 92 & 3,2563 &, 58934 \\
$\begin{array}{l}\text { Desafío } \\
\text { Recompensa }\end{array}$ & 92 & 3,3351 &, 73817 \\
\hline
\end{tabular}

Fuente: Elaboración propia con datos del estudio original.

\section{ANOVA de un factor}

A través del análisis de ANOVA de un factor se analizaron las diferencias entre las medias de las variables de interés controladas por: a) puesto en el que se desempeña y b) tiempo de trabajar en la organización.

De la Tabla 4 se desprende que los puestos de jefatura dan mayor valor a la variable "compromiso" que los otros puestos; y dicha diferencia resulta significativa. Por otra parte, el personal con más de 5 años de laborar en la institución, da mayor valor a las variables "desafío"y "recompensa". 
Tabla 4.

ANOVA de un factor según puesto desempeñado y tiempo de laborar para la organización.

\begin{tabular}{llrl}
\hline Variable & Puesto en la organización & Signo & \multicolumn{1}{l}{ Significancia } \\
Compromiso & Jefatura/Operativo & + & $0.104^{*}$ \\
& Jefatura/Otro & + & $0.064^{*}$ \\
Recompensa & Operativo/Otro & + & $0.043^{* *}$ \\
& Tiempo de estar en la Organización & & \\
Compromiso & Menos de 2 años/Más de 5 años & - & $0.035^{* *}$ \\
Desafío & De 2 a 5 años/Más de 5 años & - & $0.056^{*}$ \\
Recompensa & Menos de 2 años/Más de 5 años & - & $0.078^{*}$ \\
\hline
\end{tabular}

Nota: Significancia al * 90\%; ${ }^{* *} 95 \% ; * * * 99 \%$.

Fuente: Elaboración propia con datos del estudio original.

\section{Regresión logarítmica}

La regresión logarítmica se muestra en la Tabla 5. Las variables que presentan significancia positiva son la "comunicación", el "compromiso" y con significancia negativa las "relaciones interpersonales horizontales". Esto significa que si la organización quiere potencial y mejorar el clima organizacional debe fortalecer las acciones relacionadas con el "compromiso" y la "comunicación". Esta regresión tiene un coeficiente de determinación de 0.230.

Tabla 5

Regresión Logarítmica de las ocho variables en estudio.

\begin{tabular}{|c|c|c|c|}
\hline Variable & $\begin{array}{l}\text { Estima- } \\
\text { dor }\end{array}$ & p-value & sig. \\
\hline Constante & $-17,25$ & 0,054 & * \\
\hline Relaciones Interpersonales Horizontales & $-2,31$ & 0,076 & * \\
\hline Relaciones Interpersonales Verticales & 0,77 & 0,633 & \\
\hline Comunicación & 2,12 & 0,05 & ** \\
\hline Planificación y Coordinación & 0,42 & 0,725 & \\
\hline Empoderamiento & 2,27 & 0,121 & \\
\hline Compromiso & 2,306 & 0,004 & $* * *$ \\
\hline Desafío & $-1,093$ & 0,145 & \\
\hline Recompensa & 0,173 & 0,793 & \\
\hline
\end{tabular}

Nota: Significancia al * $90 \% ;{ }^{* *} 95 \% ;{ }^{* *} 99 \%$.

Fuente: Elaboración propia con datos del estudio original. 


\section{Conclusiones}

De los resultados del presente estudio se desprende que:

La percepción en las tres dimensiones del estudio es homogénea con una tendencia central, lo que indica que la mayoría de los funcionarios(as) dan respuestas en puntos medios denotando una percepción indiferente.

En la Municipalidad de San Rafael se requiere potenciar la comunicación y compromiso de sus colaboradores para mejorar la percepción del clima organizativo. Ambos elementos resultan fundamentales para lograr relaciones humanas adecuadas y mejorar el rendimiento en cualquier organización. Al respecto Dyer y otros (2012) afirman que los equipos de alto desempeño cuentan con integrantes con habilidades, actitudes y competencias encaminadas a lograr sus objetivos; entre ellas destacan la comunicación y resolución de problemas en una atmósfera de apoyo, confianza y compromiso.

Es importante desincentivar el valor que se da a las relaciones interpersonales horizontales (chismes, falta de transparencia, individualismo) en la percepción del clima para lograr su mejora. Dichos resultados son congruentes en el análisis cualitativo del estudio original. Fomentar elementos motivacionales de locus de control interno facilitaría dicho proceso (empoderamiento, estrategias de comunicación asertiva y promoción de la participación en la resolución de conflictos por ejemplo). Esto resulta coherente con el requerimiento de potenciar la comunicación.

Las personas con mayor tiempo de laborar en la Municipalidad muestran mayor valoración del compromiso para considerar un clima favorable. Tener tareas definidas y claras, realimentar el valor de la participación individual en el logro de las metas grupales y la motivación constante podrían ser estrategias funcionales para lograrlo.

El desafío y recompensa suelen ser variables valoradas en la percepción de un clima organizacional favorable para las personas con menos de cinco años de laborar en la organización. Identificar las competencias de los colaboradores(as) y ubicarles en puestos y tareas donde puedan potencializarlas, crear un plan de incentivos acorde a las necesidades reales, podría favorecer dicho requerimiento.

La evaluación periódica del clima permite a las organizaciones tener elementos sustantivos para priorizar la inversión y orientar acciones focalizadas al factor humano. Es recomendable que a partir de una línea base se realicen ejercicios, al menos una vez al año, que permitan 
realizar comparaciones y valorar los efectos de las acciones aplicadas con miras a mejorar el clima organizacional. La información generada permite guiar procesos relacionados con el factor humano, como capital fundamental de cualquier empresa.

\section{Discusión}

El presente artículo muestra que, emplear técnicas multivariadas permitió profundizar en los resultados del estudio de Ávila y Morera (2013); facilitando información para la priorización de dimensiones y variables para su posterior abordaje.

Se concluye que, la Municipalidad de San Rafael, para mejorar la percepción sobre su clima debe fortalecer acciones y recursos en las dimensiones de "Estructura, Procedimientos y Funciones" y"Motivación y Compromiso"; específicamente en las variables "Comunicación"y "Compromiso e Identidad"; además, contemplar que los puestos de jefatura dieron mayor valor a la variable "Compromiso" y que el personal con más de cinco años de laborar en la institución a la variable "desafío y recompensa".

Dichos resultados son congruentes con el estudio de Cardona y Zambrano (2014) donde se mencionan las dimensiones de claridad organizacional, ("Estructura, Procedimientos y Funciones") y motivación institucional como elementos comunes.

El estudio inicial describe el comportamiento de las variables clave de la Municipalidad de San Rafael y da recomendaciones para cada una de ellas, sin embargo, para los procesos de planificación económica y social, y toma de decisiones se requiere identificar una ruta, iniciando por aquellas dimensiones y variables que aportan más a la percepción sobre el clima en esa entidad particular.

Vale resaltar la necesidad de ajustar la escala de medición de la percepción utilizada e incluir una pregunta directa sobre la percepción del clima organizacional. Esto permitirá contar (potencialmente) con mayor variabilidad en las respuestas de los encuestados.

Partiendo de los resultados de este estudio y con la finalidad de aportar a la comprensión del clima organizacional, se sugiere profundizar en futuras investigaciones en:

- Comparar los resultados de las preguntas redactadas en positivo y las redactadas en negativo, con la finalidad de conocer si existen diferencias según la forma de plantearse las preguntas. 
- Analizar el peso de las variables dentro de cada dimensión para elaborar un instrumento con un indicador de clima. Dicha herramienta facilitaría identificar dicho peso de manera diferenciada según las particularidades de cada organización.

- A fin de simplificar el instrumento, resultaría importante identificar los descriptores que resultan más explicativos de las variables.

- Comparar los resultados de diferentes municipalidades en búsqueda de tendencias facilitaría la comprensión del comportamiento del clima organizacional en una institución que por su carácter híbrido (político-autónomo) podía arrojar resultados particulares.

\section{Referencias bibliográficas}

Amaro, L., González, R. y Pérez, F. (2015). Diagnóstico de Clima Organizacional y satisfacción laboral en instalaciones hoteleras. Revista Retos Turísticos, Vol. 14 No. 1.

Ávila, A., Morera I. (2013); Análisis de la percepción de los funcionarios y funcionarias de la Municipalidad de San Rafael de Heredia respecto a variables claves para optimizar el clima organizacional, en el periodo que va del 27 de mayo al 30 de setiembre del 2013; Programa CAMBIOS-EPPS-UNA. Informe final de consultoría. 270 páginas. Realizado para la Municipalidad de San Rafael de Heredia.

Brunet, L. (1992). El clima de trabajo en las organizaciones: definición, diagnóstico y consecuencias. México:Trillas.

Cardona, D. y Zambrano, R. (2014). Revisión de instrumentos de evaluación de clima organizacional. Revista Estudios Gerenciales 30 (2014) 184-189. ELSEVIER DOYMA, España.

Campbell, J., Dunnette, M., Lawler, E., \& Weick, K. (1970). Managerial behaviour, performance, and effectiveness. McGraw-Hill.

Chiang, M., Martín, M. y Núñez, A. (2010). Relaciones entre el clima organizacional y la satisfacción laboral. Volumen 2. Universidad Pontifica Comillas, Madrid.

Chiavenato, I. (2009) Comportamiento organizacional. (Segunda Edición) México, Editorial Mc Graw Hill. 
Cuadras, C. (2014) Nuevos métodos de análisis multivariante. CMC Editions. Barcelona, España. Recuperado de http://www.ub.edu/stat/personal/cuadras/metodos.pdf.

Dyer, W., Dyer, W. y Dyer, J. (2012) Equipos de trabajo de alto desempeño. Estrategias infalibles para perfeccionarlos. México, Grupo Editorial Patria.

Fischer, G. (1992). Campos de intervención en psicología social: grupo, institución, cultura, ambiente social. Volumen 124 de Educación Hoy. Narcea Ediciones. Madrid.

Franklin, E. y Krieger, M. (2011). Comportamiento Organizacional. Enfoque Para América Latina. Mexico, Editorial Pearson.

Hair, J., Black, W., Babin, B. and Anderson, R. (1995). Multivariate Data Analysis. Editorial Prentis Hall.

Halpin, A. \& Croft, D. (1962). The organizational climate of schools. Chicago: Midwest Administration Center of the University of Chicago.

Jones, G. (2013) Teoría Organizacional: Diseño y cambio en las organizaciones. (Séptima edición) México, Editorial PEARSON.

Juárez, S. (2012). Clima Organizacionaly Satisfacción Laboral. Rev. Med. Instituto Mexicano de Seguro Social 2012; 50 (3): 307-314.

Katerndahl D., Parchman, M., \& Wood, R. (2009). Perceived complexity of care, perceived autonomy, and career satisfaction among primary care physicians. Journal of the American Board of Family Medicine, 22(1): 24-33.

Koontz H., Weihrich H. y Cannice M. (2012). Administración: una perspectiva global y Empresarial. (14ªedición), México, Ed. McGraw-Hill.

Lewin, K., Lippit, R. and White, R. (1939). Patterns of aggressive behavior in experimentally crated «social climates». Journal of Social Psycology, 10, 271-299.

Likert, R. (1961). New Patterns of Management. Estados Unidos, editorial Mc Graw Hill. 279 p.

Likert, R. (1967). The human organization: Its Managmente and Value. Estado Unidos, editorial Mc. Graw Hill. 258 p.

Litwin, G. y Stringer, R. (1968). Motivation and organization climate. Cambridge: Harvard University. 
Montero, J. (2008). Análisis Multivariante. Colección manuales uex - 59. Espacio Europeo Educación Superior. Universidad de Extremadura. Servicio de Publicaciones.

Navarro-Reynoso, F., Hernández-Lamas, H., Robledo- Galván, H., \& Romero-Palacios, T. (2008). Encuesta de satisfacción del personal de cinco servicios de un hospital general. Revista CONAMED, 12(3):5-12.

Patlán-Perez J., Martínez E. y Hernández R. (2012). El clima y la justicia organizacional y su efecto en la satisfacción laboral. Revista Internacional Administración \& Finanzas, 5 (2): 1-20.

Pérez-Zapata, D., Peralta-Montecinos, J., Fernández-Dávila, P. (2014). Influencia de variables organizacionales en la calidad de vida laboral de funcionarios el sector público de salud en el extremo norte de Chile. Revista Universitas Psychologica. Vol. 13, No. 2. Págs. 15-25.

Ricarte, P. (S.F.). Relaciones en el entorno del trabajo. España, Editorial Donostiarra.

Schneider, B. (1975). Organizational climates: An essay. Personal Psychology, 28: 447-479.

Segredo A.M. (2013). Clima organizacionalen lagestión del cambio para el desarrollo de la organización. Revista Cubana de Salud Pública, Vol. 39(2), pp.385-393.

Singh, P., y Loncar, N. (2010). Antecedents of pay satisfaction in a unionized environment. Relations Industrielles/Industrial Relations, 65(3): 470-490. 